\title{
FLOW OVER TIME PROBLEM WITH INFLOW-DEPENDENT TRANSIT TIMES
}

\author{
Durga Prasad Khanal", Urmila Pyakurel, Tanka Nath Dhamala* \\ Central Department of Mathematics, Tribhuvan University, Kirtipur, Kathmandu, Nepal \\ Corresponding authors:durgapsdkhanal@gmail.com;amb.dhamala@daadindia.org
}

(Received: September 3, 2018; Revised: December 18, 2018; Accepted: December 20, 2018)

\begin{abstract}
Network flow over time is an important area for the researcher relating to the traffic assignment problem. Transmission times of the vehicles directly depend on the number of vehicles entering the road. Flow over time with fixed transit times can be solved by using classical (static) flow algorithms in a corresponding time expanded network which is not exactly applicable for flow over time with inflow dependent transit times. In this paper we discuss the time expanded graph for inflow-dependent transit times and non-existence of earliest arrival flow on it. Flow over time with inflow-dependent transit times are turned to inflow-preserving flow by pushing the flow from slower arc to the fast flow carrying arc. We gave an example to show that time horizon of quickest flow in bow graph $G^{B}$ was strictly smaller than time horizon of any inflow-preserving flow over time in $G^{B}$ satisfying the same demand. The relaxation in the modified bow graph turns the problem into the linear programming problem.
\end{abstract}

Keywords: Inflow-dependent transit times, Bow graph, Inflow-preserving flow, Quickest flow, Earliest arrival flow

\section{INTRODUCTION}

Due to increasing population and the economic activities ever day, numbers of vehicles are increasing rapidly. But limited capacity of roads causes major traffic problem in most of the cities. To solve this problem, a better traffic management system and route guidance is essential. Dynamic network flow theory, traffic simulation, models based on fluid dynamics, control theory and variational inequalities are common approaches to study the problem. Simulation optimization is the process of finding the best input variable values from among all possibilities without explicitly evaluating each possibility. The objective of simulation optimization is to minimize the resources spent while maximize the information obtained in a simulation experiment. Fluid dynamics is specially based on differential equations. It defines continuous dynamic behavior of fluids or traffic flow in small size network only. Similarly, optimal control theory is an approach to the dynamic optimization without being constrained to interior solutions. However, it calculates on differentiability.

Time is an essential component for the flow of vehicles that travel through a road network. Thus, transmission time plays an important role on the vehicle rout problems. In this paper, we focus on flow-dependent transit times for the purpose of our study. Actually, flow dependent transit times can be divided in two ways: inflow-dependent transit times and load-dependent transit times. We focused our study in inflow-dependent transit times. In inflowdependent transit times, transit time on arc only depends on the current rate of inflow in to that arc (Köhler et al. 2002). In the context of road traffic, this assumption means that the time needed to drive through a street depends on the number of cars entering the road at that moment in time. That is, transit time is a function of inflow rate. In load-dependent transit times, total amount of flow on the arc is used as input of the transit time function (Köhler \& Skutella 2005).

An important application of flow over time problem is evacuation planning problem. In continuous time setting, different dynamic network flow problems have been solved for evacuation planning problems. The continuous dynamic flow was studied and continuous contraflow models were introduced (Pyakurel \& Dhamala 2015, 2016, 2018, Dhamala \& Pyakurel 2013). Efficient algorithms were presented to solve maximum dynamic, quickest and earliest arrival contraflow problems using natural transformation of Fleischer and Tardos (1998) by reversing the direction of arcs at time zero.

This paper is structured as follows: The basic concepts and flow models were given in first section. This section was followed by flow models of flow over time with inflow-dependent transit times which is presented by fan graph and bow graph. Next section included approximation to the quickest flow problem and nonexistence of earliest arrival flow with inflow-dependent transit times. This was followed by modified bow graph and quickest inflow preserving flow. Finally the last section included concluding remarks.

\section{FLOW MODELS}

Network flows are related with directed graphs $G=(N, A)$, where $N$ stands for nodes and $A$ for arcs. Each arc $a \in A$ has positive capacity denoted by $u_{a}$ and a non-negative, left-continuous and non-decreasing transit time function $\tau_{a}:\left[0, u_{a}\right] \rightarrow \mathrm{R}^{+}$. Note that the non-decreasing function $\tau$ is left continuous if and only if $\sup \left\{\tau\left(x^{\prime}\right) \mid x^{\prime}<x\right\}=\tau(x)$. If arc $a=(v, w) \in A$ then we denote head $(a)$ for head node 
$w$ and tail $(a)$ for tail node $v$ of arc $a$. Actually, the transit time $\tau_{a}$ of an arc $a$ is the amount of time it takes to reach from the tail to the head of that arc. In general, a flow entering arc $a$ at time $\theta$ arrives at head (a) at time $\theta+\tau_{a}$. We say that any flow over time has time horizon $T$ if no flow is entering an arc $a$ after the time $T-\tau_{a}$.

Similarly, the transit time of a path $P$ in $G$ with static flow $x$ is defined as $\tau_{p}(x)=\sum_{a \in P} \tau_{a}\left(x_{a}\right)$. In case of constant transit time of a path $P$, it is simply denoted by $\tau_{P}$. Source nodes has supply $D \geq 0$ and sink node $t$ has demand $D \leq 0$. Nodes that are neither source nor sink are called intermediate nodes. For the node $v$, we denote $\delta^{+}(v)$ and $\delta^{-}(v)$ for the set of arcs leaving and entering the node $v$ respectively. Sometimes we consider flows with costs. Then, each arc $a \in A$ has associated cost coefficients $c_{a} \geq$ 0 , where $c_{a}$ is interpreted as the cost per flow unit for sending through the arc.

Static network flow: A static network flow $x: A \rightarrow \mathbf{R}^{+}$in $\mathrm{G}$ assigns to every arc $a$, a non-negative flow value $x_{a}$ which satisfies the flow conservation

$$
\sum_{a \in \delta^{+}(v)} x_{a}-\sum_{a \in \delta^{-}(v)} x_{a}=0, \quad \text { for all } v \in N \backslash\{s, t\} .
$$

The static flow $x$ is called feasible, if it satisfies the capacity constraints $0 \leq x_{a} \leq u_{a}$, for all arcs $a \in A$. The value of an $s$ - $t$ flow $x$ is defined as

$$
|x|=\sum_{a \in \delta^{+}(s)} x_{a}-\sum_{a \in \delta^{-}(s)} x_{a}=0 .
$$

In case of the flow with cost, the cost of a static flow $x$ is defined as $c(x)=\sum_{a \in A} c_{a} x_{a}$.

Continuous flow over time: The continuous flow over time $f$ in $G$ is a Lebesgue measurable function $f_{a}: A \times \mathbf{R}^{+}$ $\rightarrow \mathbf{R}^{+}$, for every $a \in A$. Here, $f_{a}(\theta)$ is the rate of flow per unit time that enters arc $a$ at time $\theta$. Clearly $f_{a}(\theta)=0$ for $\theta<0$. If the flow is allowed to storage at intermediate nodes, we write it as

$$
\sum_{a \in \delta^{+}(v)} \int_{0}^{\zeta} f_{a}(\theta) d \theta-\sum_{a \in \delta^{-}(v)} \int_{\tau_{\alpha}}^{\zeta} f_{a}\left(\theta-\tau_{a}\right) d \theta \leq 0,
$$

for all $\zeta \in[0, T)$ and $v \in N \backslash\{s, t\}$. An equality holds for $v \in N \backslash\{s, t\}$ at time $\zeta=T$. The flow $f$ is said to be feasible if $0 \leq f_{a}(\theta) \leq u_{a}$ for all $\theta \in \mathbf{R}^{+}$and $a \in A$. The flow over time $f$ satisfies supplies and demands if

$$
\sum_{a \in \delta^{\circ+(v)}} \int_{0}^{T} f_{a}(\theta) d \theta-\sum_{a \in \delta=(v)} \int_{\tau_{a}}^{T} f_{a}\left(\theta-\tau_{a}\right) d \theta=D,
$$

for every $v \in\{s, t\}$. The value of $s$ - $t$ flow over time $f$ is given by

$$
|f|=\sum_{a \in \delta^{+}(s)} \int_{0}^{T} f_{a}(\theta) d \theta-\sum_{a \in \delta^{-}(s)} \int_{\tau_{a}}^{T} f_{a}\left(\theta-\tau_{a}\right) .
$$

Here, $|f|$ is the total amount of flow leaving the source node $s$ until time $T$. Due to flow conservation, this value is equals to the total amount of flow arriving in the sink node $t$ until time $T$. The cost of $s$ - $t$ flow over time $f$ is defined as $c(f)=\Sigma_{a \in A} c_{a} \int_{0}^{T} f_{a}(\theta) d \theta$.

Temporally repeated flow: Let $x$ be a feasible static flow over time in $G$ with path decomposition $\left(x_{p}\right)_{p \in P}$, where $P$ is a set of paths such that the transit time $\tau_{p}$ of every path $p$ $\in P$ is bounded above by $T$. The temporally repeated flow $f$ with transit time $\left(\tau_{a}\right)_{a \in \mathrm{A}}$ and time horizon $T$, as in (Hall et al. 2003a, 2003b), is defined as follows:

1. For every path $p \in P$, flow $f$ enters path $p \in P$ at constant rate $x_{p}$ starting at time zero and ending at time $\quad T-\tau_{p}(x)$.

2. The transit time of every arc $a \in A$ is fixed to $\tau_{a}$, i.e., at every point in time $\theta \in[0, T)$, flow units entering arc $a$ at time $\theta$ reaches head ( $a$ ) at time $\theta+\tau_{a}$.

The value of a temporally repeated $s$ - $t$ flow $f$ with underlying static flow $\left(x_{p}\right)_{p \in P}$ is given by

$$
|f|=\sum_{p \in P}\left(T-\tau_{p}\right) x_{p}=T|x|-\sum_{a \in A} \tau_{a} x_{a} .
$$

Discrete flow over time: Assume that all transit times are integral values. A discrete flow over time $f$ in $G$ assigns to every arc $a \in A$, a function $f_{a}: A \times \mathbf{Z}^{+} \rightarrow \mathbf{R}^{+}$. We say that the flow over time $f$ has time horizon $T$, if no flow is entering an arc $a$ after the time $T$-1- $\tau_{a}$ i.e., $f_{a}(\theta)=0$ for all $\theta \geq T-\tau_{\alpha,}, a \in A$. Flow conservation constraints are as similar to the continuous flow over time except the integral over time is replaced by the sum. It is modeled as

$$
\sum_{a \in \delta^{+}(\mathrm{v})} \sum_{\theta=0}^{\zeta} f_{a}(\theta)-\sum_{a \in \delta^{-}(\mathrm{v})} \sum_{\theta=\tau_{\alpha}}^{\zeta} f_{a}\left(\theta-\tau_{a}\right) \leq 0,
$$

for all $\zeta \leq T-1$ and $v \in N \backslash\{s, t\}$. Also the equality holds for $v \in N \backslash\{s, t\}$ at time $T$-1. The flow $f$ is said to be feasible if $0 \leq f_{a}(\theta) \leq u_{a}$ for all $\theta \in \mathbf{Z}^{+}$and $a \in A$. The discrete flow $f$ satisfies supplies and demands if

$$
\sum_{a \in \delta^{+}(\mathrm{v})} \sum_{\theta=0}^{T-1} f_{a}(\theta)-\sum_{a \in \delta} \sum_{\theta=\tau_{\alpha}}^{T-1} f_{a}\left(\theta-\tau_{a}\right)=D,
$$

for every $v \in\{s, t\}$. The cost of a discrete flow over time $f$ is defined as $c(f)=\sum_{a \in A} c_{a} \sum_{\theta=0}^{T-1} f_{a}(\theta)$.

Time expanded graph: For a graph $G=(N, A)$ with integral transit times on arcs and integral time horizon $T$, the $T$-time expanded graph of $G$, denoted by $G(T)$, is obtained by creating $T$ copies of $N$ which are labeled as 
$N(0), N(1), \ldots ., N(T-1)$ together with $\theta^{\text {th }}$ copy of node $\mathrm{v}$ denoted by $\mathrm{v}(\theta), \quad \theta \in\{0,1, \ldots, T-1\}$. For every arc $a=$ $(v, w) \in A$ and $0 \leq \theta<T-\tau_{a}$, there is an arc $a(\theta)$ from $v(\theta)$ to $w\left(\theta+\tau_{a}\right)$ with the same capacity as arc $a$. If the storage of flow at node $v \in N$ is allowed, we include an infinite capacity holdover arc from $v(\theta)$ to $v(\theta+1)$ for all $0 \leq \theta<$ $T$-1, which models the possibility to hold flow at node $v$. Figure 1(b) represents time expansion of Fig. 1(a) for time horizon $T=6$. Upward holdover arcs are included in time expanded graph, if storage at intermediate nodes is allowed, otherwise they are omitted.

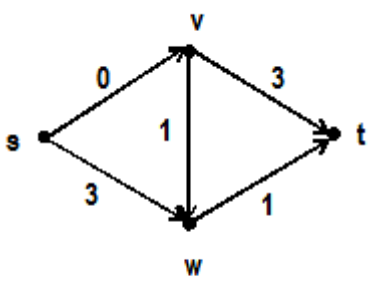

(a)

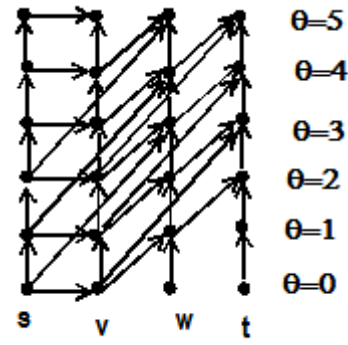

(b)
Fig. 1. (b) represents the T-time expansion of network (a) for time horizon $\mathrm{T}=6$

\section{Flow-dependent transit times}

Inflow-dependent transit times: The main objective of this section was to study about flow over time with inflow-dependent transit times, which is an extension of the flow over time with fixed transit times. Transit times are fixed in flow over time with fixed transit times so that the flow on arc $a$ progresses at constant speed. Here, in inflow-dependent transit times (Köhler et al. 2002), transit times experienced by an infinitesimal unit of flow on an arc is determined when entering this arc and only depends on the inflow rate at that moment of time. In the flow over time with inflow-dependent transit times, flow entering arc $a$ at time $\theta$ arrives head $(a)$ at time $\theta+$ $\tau_{a}\left(f_{a}(\theta)\right)$. In particular, the transit time of an arc only depends on the current flow rate. In time dependent flow, all arc must be empty from time $T$ on, so for all arcs $a \in A$ and $\theta \in \mathbf{R}^{+}$we have $\theta+\tau_{a}\left(f_{a}(\theta)\right)<T$ whenever $f_{a}(\theta)>0$. Flow conservation, in this case, is modeled as

$$
\sum_{a \in \delta^{+}(v)} \int_{0 \leq \theta<\zeta} f_{a}(\theta) d \theta-\sum_{a \in \delta^{-}-(v)} \int_{\theta \geq 0: \theta+\tau_{a}\left(f_{a}(\theta)\right) \leq \zeta} f_{a}(\theta) d \theta \leq 0,(9)
$$

for all $\zeta \in[0, T)$ and $v \in N \backslash\{s, t\}$ and equality holds for $v \in N \backslash\{s, t\}$ at time $\zeta=T$. The flow over time $f$ satisfies the supply and demands if

$$
\sum_{a \in \delta^{+}(v)} \int_{0 \leq \theta<\zeta} f_{a}(\theta) d \theta-\sum_{a \in \delta^{-}(v)} \int_{\theta \geq 0: \theta+\tau_{a}\left(f_{a}(\theta)\right) \leq \zeta} f_{a}(\theta) d \theta=D,(10)
$$

for $v \in\{s, t\}$. The value of $s$ - $t$ flow over time $f$ is given by

$$
|f|=\sum_{a \in \delta^{+}(s)} \int_{0}^{T} f_{a}(\theta) d \theta-\sum_{a \in \delta^{-}(s)} \int_{\tau_{a}}^{T} f_{a}(\theta) d \theta .
$$

The value of a temporally repeated flow $f$ with flowdependent transit times $\left(\tau_{a}\right)_{a \in \mathrm{A}}$ and underlying path decomposition $\left(x_{p}\right)_{p} \in \mathbf{P}$ is given by

$$
|f|=\sum_{p \in P}\left(T-\tau_{p}\left(x_{p}\right)\right) x_{p}=T|x|-\sum_{a \in A} \tau_{a}\left(x_{a}\right) x_{a} .
$$

Fan graph: Mostly flow over time problems can be solved by static flow algorithms in time expanded graphs. As defined in Köhler et al. (2002), fan graph $G^{F}$ is generalized time-expanded graph, in which transit time indirectly depends on the current flow rate. To focus the step function character of transit time function, it is denoted here by $\tau_{a}^{s}$ instead of $\tau_{a}$. To compute the flow over time with inflow-dependent transit times in $G$, classical network flow algorithm for static flow problem is applied in $G^{F}$.

As defined in time expanded graph, fan graph $G^{F}$ is also defined on the same set of nodes $\left\{v_{\theta}: v \in N_{v} \theta=0,1\right.$, ...., $T-1\}$. For every $\theta \in\{0,1, \ldots ., T-1\}$, we define a fan of arcs leaving $v_{\theta}$, which represents all possible transit times of arc $a=(v, w)$ and is expanded according to the transit time function. For example, take a single arc $a=(v, w)$ with transit time $\tau_{a}$ as 1, 3 and 7 if the flow is at most 2, 4 and 6 units, respectively, as shown in Fig. 2(a). Figure 2(b) shows the fan at $\theta=0$ consisting capacitated horizontal arcs and uncapacitated arcs pointing upward. Similarly, Figure 2(c) shows the fan graph of arc $a$ as a time expanded graph having infinite capacity from $v_{\theta}$ to $v_{\theta+1}$ for all $v \in N$ and $\theta=0,1, \ldots . ., T-2$, which allow to hold flow at a node $v$.

Although choice of transit times and capacities are according to the given transit time functions, the transit time experienced in the fan graph do not completely reflects inflow-dependent transit times. In this example, if the flow is entering arc $a$ at constant rate 6 , then by transit time function, every 6 unit flow should traverse arc $a$ with transit time $\tau_{a}=7$. But in the fan graph, first 2 unit flows traverse the arc with transit time 1 , another 2 units with transit time 3 and rest 2 units with transit time 7 . Hence only last 2 unit flow is experiencing the correct transit time 7 .

Bow graph: As described in Köhler et al. (2002), the bow graph denoted by $G^{B}=\left(N^{B}, A^{B}\right)$, arises from the original graph by expanding arc $a \in A$ according to its transit time function. In bow graph $G^{B}$, every arc $e \in A^{B}$ has capacity $u_{e}$ and a constant transit time $\tau_{e} \in \mathrm{R}^{+}$. For the definition, let us consider an arc $a \in A$ with capacity $u_{a}$ with break points $0=u_{0}<u_{1}, \ldots . .<u_{m}=u_{a}$ and 
corresponding transit times $\tau_{1}<\tau_{2}<\ldots .<\tau_{m}$, where $\tau_{a}^{s}(x)=\tau_{i}$ for $x \in\left(u_{i-1}, u_{i}\right]$. This means that flow entering the arc $a$ at the rate $x \in\left(u_{i-1}, u_{i}\right.$ ] needs $\tau_{i}$ time unit to traverse arc. To construct bow graph of an arc $a$, we replace it by arcs of two types: bow arcs denoted by $b_{1}, b_{2}, \ldots, b_{m}$ and regulating arcs denoted by $r_{1}, r_{2}, \ldots$ ,$r_{m}$. The bow arcs are uncapacitated, and they represent all possible transit times of arc $a$. The transit times of bow arc $b_{i}$ is given by $\tau_{i}, i=1,2, \ldots, m$. The regulating arcs have zero transit time and they limit the amount of flow entering the bow arcs. Their capacities are chosen

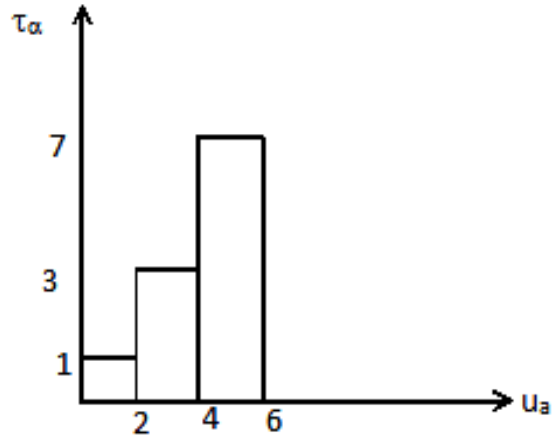

(a)

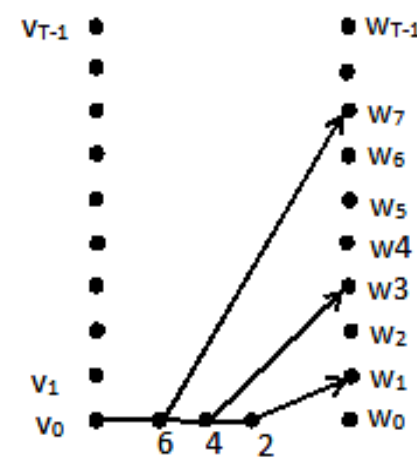

(b) according to the breakpoints of transit time function $\tau_{a}^{s}\left(x_{a}\right)$, that is, the capacity of arc $r_{i}$ is set to $u_{i}, i=1,2, \ldots$, $m$. The set of regulating arcs and bow arcs of an $\operatorname{arc} a \in A$ is denoted by $A_{a}^{B}$. For every arc $e \in A_{a}^{B}$, we denoted by $a(e)$ as the corresponding $\operatorname{arc} a$ in $A$. The size of $A_{a}^{B}$ is linear in the number of breakpoints of $\tau_{\llbracket}^{s}$. In bow graph, nodes in $N^{B}$ corresponding to nodes in $N$ are original nodes and the remaining nodes are artificial. Figure 3(b) represents the expansion of an arc $a$ according to transit time function using bow graph in Fig. 3. Note that fan graph is the time-expanded graph of the bow graph.
Fig. 2. An expansion of single arc $a=(v, w)$ with given transit times by definition of fan graph

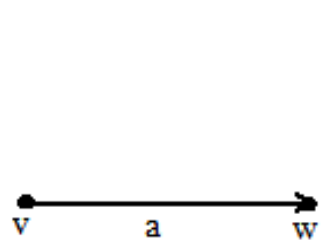

(a)

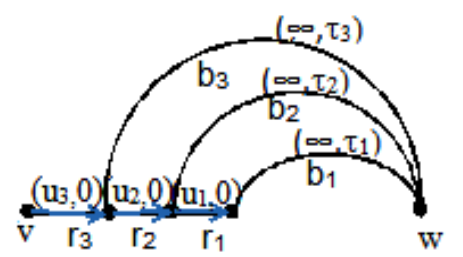

(b)
Fig. 3. An expansion of single arc $a=(v, w)$ according to transit time function by definition of bow graph

Relaxation: Flow entering arc $a$ at time $\theta$ and traversing the arc $a$ with transit time $\tau_{i}$ in the original graph $G$ also enters the expansion of arc $a$ in $G^{B}$ at time $\theta$ and traverses in time $\tau_{i}$, using the corresponding bow arc $b_{i}$. Let $f$ be a flow over time in $G$ with inflow-dependent transit times $\left(\tau_{\square}^{s}\right)_{a \in A}$ and time horizon $T$. Then, it can be interpreted as a flow over time with constant transit times $f^{B}$ with the same time horizon $T$ in $G^{B}$. According to the transit time function, flow entering the arc $a$ at time $\theta$ in the original graph $\mathrm{G}$ with flow rate $f_{a}(\theta)$ reaches head $(a)$ at time $\theta+\tau_{a}^{s}\left(f_{a}(\theta)\right)$. We use the similar behavior in the bow graph by sending flow onto the arc $e \in A_{a}^{B}$ with transit time $\tau_{a}^{s}\left(f_{a}(\theta)\right.$.

For bow $\operatorname{arcs} b_{1}, \ldots ., b_{m}$ in $A_{a}^{B}$, let $i \in\{1,2, \ldots, m\}$ be chosen such that $\tau_{a}^{s}\left(f_{a}(\theta)\right)=\tau_{i}$. We define a flow over time $f^{B}$ on the expansion of arc $a$ by setting $f_{e}{ }^{B}(\theta)=$ $f_{a}(\theta)$ if $e$ is either a bow arc $b_{i}$ or a regulating arcs $r_{j}$ with $j \geq i$. For all other $\operatorname{arcs}$ we set $f_{e}{ }^{B}(\theta)=0$. That is,
$f_{\theta}^{B}(\theta)= \begin{cases}f_{\alpha}(\theta) & \text { if } e=b_{i} \text { or } \theta \in\left\{r_{j} \mid i \leq j \leq m\right\} \\ 0 & \text { otherwise }\end{cases}$

Clearly, $f^{\bar{B}}$ obeys capacity constraints and flow conservation at all intermediate nodes. From the definition of bow graph, we have the following conclusion: Let $f$ be a flow over time with inflow-dependent transit times $\left(\tau_{a}^{s}\right)_{a \in A}$ in G that sends D units of flow from s to $t$ within the time $T$. Then flow over time $f^{B}$ in $G^{B}$ (with constant transit times) sends $D$ units of flow from s to $t$ within time $T$. This means that every flow over time in $G$ can be regarded as a flow over time in $G^{B}$ but the converse may not be true. The reason is that, in flow-dependent transit times flow entering the arc at the same time arrives the head of arc simultaneously but in bow graph flow allowed to split up along the bows of different transit times. So only a portion of flow experience the correct transit time prescribed by given transit time function. We can state this property as follows: If $x^{B}$ sends flow along the $i^{\text {th }}$ bow arc $b_{i}$ with index $\mathrm{j} \leq i$, we have $x_{b_{j}}^{B}=u_{j}-u_{j-1}$. That is, the flow $x^{B}$ fills the bows from bottom to top. This is because, if $\mathrm{j} \leq i$, the transit time of arc $b_{j}$ is smaller than the transit time of arc $b_{i}$. Therefore, shifting flow from $b_{i}$ to $b_{j}$ improves the value of the objective function of $x^{B}$.

Definition (Inflow-preserving flow): Let $f^{B}$ be a flow over time in $G^{B}$. Then flow $f^{B}$ is inflow-preserving if for every original arc $a \in A$ and at every point in time $\theta$, the flow $f^{B}$ sends flow into at most one bow arc e $\in A_{a}^{B}$. 
Now by definition of inflow-preserving flow, we have the following fact: Every inflow-preserving flow over time $f^{B}$ in $G^{B}$ with time horizon $T$ corresponds to a flow over time $f$ in $G$ with inflow-dependent transit times $\left(\tau_{a}^{a}\right)_{a} \in_{A}$ and time horizon $T$, and vice versa. It is to be noted that the time horizon of quickest flow in $G^{B}$ can be strictly smaller than time horizon of any inflow-preserving flow over time in $G^{B}$ satisfying the same demand.

Example 1: Let $G$ consists of a path $P$ of length 2 denoted by $a_{1}$ and $a_{2}$ with respective capacities $u_{a_{1}}=2$ and $u_{a_{2}}=2$. The first arc on $P$ has transit time function

$\tau_{a_{1}}^{s}(x)= \begin{cases}0 & \text { if } 0 \leq x \leq 1 \\ 1 & \text { if } 1<x \leq 2\end{cases}$

The second arc has constant transit time $\tau_{a_{2}}=0$ (Fig. 4).

There exist a flow over time in $G^{B}$ which sends $D=9$ units of flow from $s$ to $t$ within given time $T=5$. For, using temporally repeated flow which sends flow at rate 1 into the $s-t$ path containing lower bow arc $b_{1}$ during time interval $(0,5)$ and flow rate 1 into the $s$ - $t$ path containing upper bow arc $b_{2}$ during time interval $(0,4)$. We define an inflow-preserving flow over time $f$ in $G^{B}$ which satisfies demand 8 within time $T=5$. It sends flow at rate 2 into bow arc $b_{2}$ during $(0,3)$, then after it sends flow at the rate 1 into bow arc $b_{1}$ until time 5 .

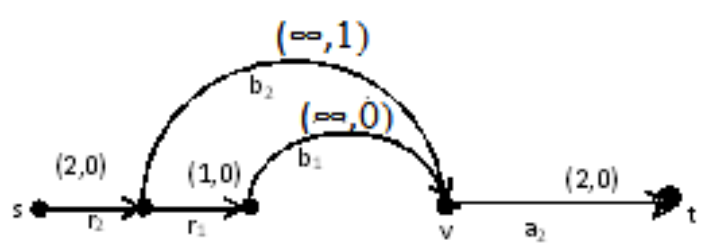

Fig. 4. Bow graph according to the transit time function of example 1

\section{Approximation to the quickest flow problem}

Quickest inflow-dependent flow problem is to determine an $s$ - $t$ flow over time with inflow-dependent transit times that satisfies demand $D$ within minimum time $\bar{T}$.

Ford and Fulkerson (1958) assumed $\bar{T}^{B}$ be the minimum time horizon and $x^{B}$ be a static flow in $G^{B}$ such that the value of the resulting temporally repeated flow $f^{B}$ in $G^{B}$ is

$\left|f^{\bar{B}}\right|=\bar{T}^{\bar{B}}\left|x^{\bar{B}}\right|-\sum_{\epsilon \in A^{B}} \tau_{\varepsilon} x_{\varepsilon}^{\bar{B}}=D$.

Since the quickest flow problem on $G^{B}$ can be seen as a relaxation of the quickest flow problem with inflowdependent transit times on $G$, the value $\bar{T}^{B}$ is the lower bound on the optimal time horizon $\bar{T}$ in $G$.
Now we are interested in a flow over time in $G^{B}$, which is inflow-preserving. As discussed above flow $f^{B}$ in $G^{B}$ in general does not satisfy this condition. One of the reason is that flow unit entering the expansion $A_{a}^{B}$ of $a \in A$ simultaneously might experience different transit time in $f^{B}$ due to different transit times on bow $\operatorname{arc} A_{a}^{B}$. So, the static flow $x^{B}$ of an arc $A_{a}^{B}$ is to be restricted make sure that it does not split among the bow arcs representing different transit times. For this, we use to push the flow from fast bow arcs up to the slowest flow carrying bow $\operatorname{arcs}$ in $x^{B}$. The modified push flow $\hat{x}^{B}$ is defined as

$\hat{x}_{b_{i}}^{B}= \begin{cases}x_{y_{i}}^{B} & \text { if } x_{b_{i}}^{B}>0 \text { and } x_{b_{j}}^{B}=0 \text { for all } j>i \\ 0 & \text { otherwise }\end{cases}$

$\hat{x}_{Y_{i}}^{B}= \begin{cases}x_{\sigma_{i}}^{B} & \text { if } x_{b_{j}}^{B}=0 \text { for all } j>i \\ 0 & \text { otherwise }\end{cases}$

In bow graph $G^{B}$, only regulating arcs $r_{1}, \ldots \ldots ., r_{m}$, are capacitated, it follows from the feasibility of $x^{B}$ in $G^{B}$ that $\hat{x}^{B}$ is feasible as well. Similarly value of the flow remains unchanged, that is, $\left|\hat{x}^{B}\right|=\left|\mathrm{x}^{\mathrm{B}}\right|$. We denote the unique bow arc $b \in A_{a}^{B}$ which carries flow in $\hat{x}^{B}$ by $\mathrm{b}^{\mathrm{a}}$ and flow decomposition of $\hat{x}^{B}$ into flows on $s-t$ path $\mathrm{p} \in \widehat{\mathrm{P}}$ in $\mathrm{G}^{\mathrm{B}}$ with flow value $\hat{x}_{\mathrm{p}}^{B}$. Thus from the construction of modified push flow we observe the following: Let $a \in A$, either no flow in $\hat{x}^{B}$ is routed through $A_{\mathrm{g}}^{\mathrm{B}}$ or the flow in $\hat{x}^{B}$ is routed through a unique bow arc $b^{\mathbb{a}} \in A_{a}^{\mathrm{B}}$ with transit time $\tau_{b^{a}}=\tau_{a}^{s}\left(\hat{x}_{b^{\mathrm{z}}}^{\mathrm{B}}\right)$. In Fig. 5, flows in bow arcs of Fig. 5(a) are pushed in single bow arc of Fig. 5(b) using definition of modified push flow.

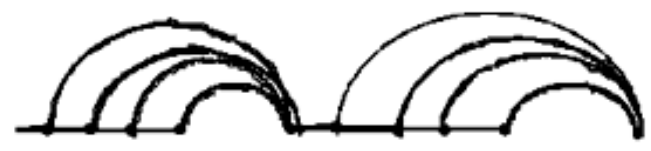

(a)

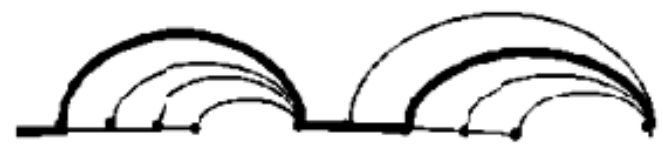

(b)

Fig. 5. (b) represents the inflow preserving temporally repeated flow of origin bow flow of (a)

Since all flow-carrying arcs $\hat{x}^{B}$ carry flow in $\mathrm{x}^{\mathrm{B}}$ as well so the transit time $\tau_{\mathrm{P}}$ of every path $\mathrm{p} \in \widehat{\mathrm{P}}$ is bounded from above by the time horizon $\bar{T}^{B}$ of a quickest flow in $G^{B}$. Now the path decomposition $\left(x_{\mathrm{p}}^{B}\right)_{\mathrm{peF}}$ of $\hat{x}^{B}$ induces a temporally repeated flow $f^{B}(T)$ in $G^{B}$ for any time horizon $T \geq \bar{T}^{E}$. We choose $\hat{T}$ such that $\left|\hat{f}^{B}(\hat{T})\right|=\hat{T}\left|\hat{x}^{B}\right|-\sum_{e \in A^{B}} \tau_{e} \hat{x}_{\theta}^{B}=D$. It is very essential to note that the flow over time $f^{B}$ is inflow-preserving. 
Lemma1: The value $\hat{T}$ is bounded from above by $2 \bar{T}^{B}$.

Proof: Since $\left|f^{B}(T)\right|$ is an increasing function of T. So it is suffices to show that $\left|\hat{f}^{B}\left(2 \hat{T}^{B}\right)\right| \geq D$.

$$
\begin{aligned}
\left|\hat{f}^{B}(2 \bar{T})\right|= & 2 \bar{T}^{B}\left|\hat{x}^{B}\right|-\sum_{\theta \in A^{B}} \tau_{\varepsilon} \hat{x}_{\theta}^{B} \\
= & 2 \bar{T}^{B}\left|\hat{x}^{B}\right|-\sum_{p \in P^{B}} \tau_{p} \hat{x}_{p}^{B} \\
& =\bar{T}^{B}\left|\hat{x}^{B}\right|+\sum_{p \in P^{P}}\left(\bar{T}^{B}-\tau_{p}\right) \hat{x}_{p}^{B} \\
\geq & \bar{T}^{B}\left|\hat{x}^{B}\right|=\bar{T}^{B}\left|x^{B}\right| \geq\left|f^{B}\right|
\end{aligned}
$$

\section{Non-existence of earliest arrival flows}

Earliest arrival flows are those maximum $s$ - $t$ flows over time that sends, for each time $\theta \in[0, T)$, the maximum amount of flow from $s$ to $t$. Although maximum flow for a fixed time horizon $T$ exists, it may not be true for each $\theta \in[0, T)$.

Gale (1959) showed the existence of earliest arrival flows for general networks with constant transit times on the arcs and, more generally, for networks with timedependent (but not flow-dependent) transit times and capacities on the arcs. In case of flow-dependent transit times there exists an $s$ - $t$ flow over time that sends the maximum amount of flow from $s$ to $t$ for any time horizon $T$. But there may not be such a maximum $s$ - $t$ flow that is maximal for each $\theta \in[0, T$ ) (Baumann \& Köhler 2007). We will show this by the following simple example.

Example 2: Consider the one-arc network together with the simple linear transit time function given by $\tau(x)=2 x$ for $0 \leq x \leq 2$ (Fig. 6) and a capacity two.

We consider a flow model with inflow-dependent transit times. Let $T=6$ be considered time horizon. When sending flows from $s$ to $t$ at a flow rate of 2 in time interval $(0,2)$ and at flow rate linearly decreasing from 2 to 0 in the time interval $(2,6)$, then the flow of 8 units has been reached to the sink $t$ by time $T=6$. In fact, this is the maximum amount of flow that can be sent from $s$ to $t$ in this time horizon.

s

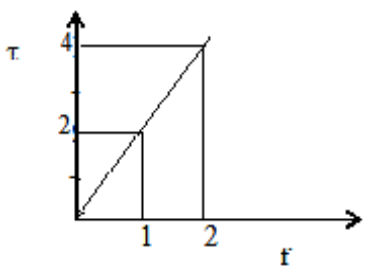

Fig. 6. Dynamic network with inflow-dependent transit times as in example 2

To construct an earliest arrival flow, we have to make sure that the maximum possible amount of flow has reached the sink for any $\theta \in(0, T)$. To show that this is not possible for this example we examine just two values of $\theta$. Sending flow at the flow rate linearly decreasing from 2 to 0 in time interval $(0,4)$ shows that an earliest arrival flow must send at least 4 units of flow to $t$ up to time $\theta=4$. In fact, sending any flow in this time interval at a higher flow rate would result in a decrease of the flow value reaching t up to time $\theta=4$. It follows easily that any flow sending the maximum amount of flow up to $\theta=4$ into $t$ cannot send more than 5 units of flow into $t$ up to $\theta=6$. However, the maximum flow for time horizon 6 is 8 . Thus we have the following conclusion:

Theorem 1: For inflow-dependent transit times, earliest arrival flow does not exist in general.

\section{The modified bow graph}

The modified bow graph, denoted by $G^{B}=\left(N^{B}, A^{B}\right)$, is defined on the same node set as G, i.e., $N^{B}=N$, and is obtained by creating several copies of an arc, one for possible transit times $\tau_{a}^{g}$ on the arc as described in previous section. Thus arc $a$ is replaced by creating $\mathrm{m}$ parallel bow arcs $b_{1}, b_{2}, \ldots ., b_{m}$. The transit time of bow arc $b_{j}$ is $\tau_{j}$ and capacity $u_{j}$ for $j=1,2, \ldots, m$ (Fig. 7). We denote the set of bow arcs corresponding to arc $a$ by $A_{a}^{B}$ and refer to $A_{a}^{B}$ as the expansion of arc $a$. The cost coefficient to every arc $e \in A_{a}^{B}$ are identical to those of arc $a$, i.e., $c_{e}=c_{a}$. For every arc $e \in A_{a}^{B}$, let $a(e)$ denote the original arc $a$. The main difference between this modified bow graph and previously defined bow graph is as follows: In the modified model, we omit the regulating arcs which, in the previous model, limit the amount of flow entering the bow arcs. In particular, all bow arcs representing the same original arc share capacity. In the modified model, capacities are directly assigned to the bow arcs. They no longer share capacities. Moreover, we include arc costs in the modified model.

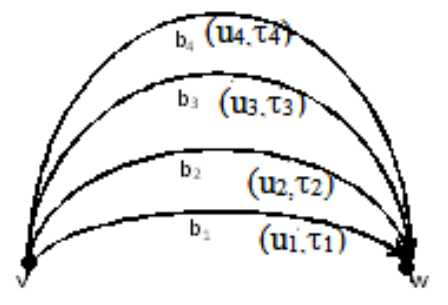

Fig. 7. An expansion of single arc $a=(v, w)$ according to modified blow graph

Relaxation: In this section, we discuss the relationship between flows over time in the bow graph $G^{B}$ and flows over time with inflow-dependent transit times in $G$. Any flow over time $f$ with inflow-dependent transit times $\left(\tau_{\llbracket}^{g}\right)_{a \in A}$ in $G$ with time horizon $T$ and cost $C$ can be interpreted as a flow over time $f^{B}$ (with constant transit times) in $G^{B}$ with same time horizon $T$ and cost $C$ as 
follows: If, in the original graph $G$, flow is entering $\operatorname{arc} a$ $\in A$ at time $\theta$ with flow rate $f_{a}(\theta)$, then, in the bow graph this flow is sent onto the bow arc $e \in A_{a}^{B}$ representing transit time $\tau_{a}^{s}\left(f_{a}(\theta)\right)$. Costs are preserved, since the cost of every arc $e \in A_{a}^{B}$ is identical to the cost of arc $a$. Unfortunately, an arbitrary flow over time $f^{B}$ in $G^{B}$ does not corresponds to a flow over time $f$ with inflowdependent transit times $\left(\tau_{a}^{s}\right)_{a \in A}$ in $G$. In addition, we define inflow-preserving property in $G^{B}$ which helps to justify the following observation: Every inflowpreserving flow over time $f^{B}$ in $G^{B}$ with time horizon $T$ corresponds to a flow over time $f$ in $G$ with inflowdependent transit times $\left(\tau_{\square}^{s}\right)_{a} \in A$ and time horizon $T$, and vice versa.

Notice that the set of inflow-preserving flows over time is not convex. In particular, it is difficult to compute inflowpreserving flows directly. Therefore, we consider a relaxed notation which can be interpreted as convexification of inflow-preserving flows and defined as follows: For any bow arc $e \in A^{B}$, let $\lambda_{e}(\theta)=f_{e}^{B}(\theta) / u_{e}$ denote the per capacity inflow rate into $\operatorname{arc} e$.

Definition (Weakly inflow-preserving): A flow over time $f^{\bar{B}}$ with time horizon $T$ in $G^{B}$ is called weakly inflow-preserving if $\sum_{\theta \in A_{a}^{B}} \lambda_{\theta}(\theta) \leq 1$ for all $a \in A$ and $\theta$ $\in[0, T)$.

\section{Quickest weakly inflow-preserving flows}

Fleischer and Skutella (2002) propose a $(2+\varepsilon)-$ approximation algorithm for the quickest multicommodity flow problem with bounded cost and constant transit times. The method based on an approximate length bounded static flow computation. The same approach can be applied to the problem of finding a quickest weakly inflow-preserving flow over time with bounded cost in the bow graph. Here the goal is to determine a weakly inflowpreserving flow over time in $G^{B}$ which satisfies all demands within minimum time $T$ at a cost bounded by $C$.

Let $f^{\bar{B}}$ be an optimal solution to this problem with minimum time horizon $T$. Consider the static flow $\mathrm{x}^{\mathrm{B}}$ in $G^{B}$ which results from averaging the flow $f^{B}$ on every arc $\mathrm{e} \in A^{B}$ over the time interval $[0, T)$, i.e $x_{\theta}^{B}=\frac{1}{T} \int_{0}^{T} f_{e}^{B}(\theta) d \theta$. As proven by Fleischer and Skutella (2005), this static flow

1. satisfies a fraction of $1 / T_{T}$ of the demands covered by the flow over time $f^{B}$,

2. has cost $c\left(x^{B}\right)=\frac{c\left(f^{B}\right)}{T}$ and

3. is $T$-length-bounded.

The later property means that the flow can be decomposed into a sum of flows on $s$ - $t$ paths such that the length $\tau(p)$ $=\Sigma_{\varepsilon \in p} \tau_{e}$ of any such path $p$ is at most $T$. To see this, notice that the flow over time $f^{B}$ finishes by time $T$, every (infinitesimal) unit of flow in $f$ describes a path in $G^{B}$ of length at most $T$. Taking all such paths yields a $T$-lengthbounded path decomposition of $x^{B}$.

Since $f^{B}$ is weakly inflow preserving, so is $x^{B}$, i.e., it's per capacity flow values $\lambda_{e}=x_{e}^{B} / u_{e}, e \in A^{B}$, satisfies

4. $\sum_{e \in A B} \lambda_{e} \leq 1$ for every arc $a \in \mathrm{A}$.

Any arbitrary static flow $\bar{x}^{B}$ in $G^{B}$ satisfying requirements (1)-(4) can be turned into a weakly inflow- preserving flow over time $g^{B}$ in $G^{B}$ meeting the same demands at the same cost as $f^{B}$ within time $2 T$ as follows: Send flow into every $s$ - $t$ path $p$ given by the length-bounded path decomposition of $x$ at the corresponding flow rate $x_{p}$ for exactly $T$ time units, wait for at most another $T$ time units until all flow has arrived at its destination. Since $g_{e}^{E}(\theta) / u_{e}$ is always upper-bounded by $x_{e} / u_{e}$, it follows from property (4) that $g^{B}$ is weakly inflow-preserving. Provided that we know the optimal time horizon of a quickest weakly inflow-preserving flow, we can compute 2-approximate solution by solving the static flow problem defined by requirements (1)-(4).

Unfortunately, computing T-length-bound flows is NPhard. Yet, as discussed in (Fleischer \& Skutella 2002), the $T$-length-bounded multi-commodity flow problem can be approximated within arbitrary precision in polynomial time by slightly relaxing the length bound $T$. It is easy to generalize this observation to length-bounded, weakly inflow-preserving flows. Let $P^{T}$ be the set of all $s$ - $t$ paths in $G^{B}$ whose transit times are bounded from above by $T$. The cost of path $p \in P$ is defined as $c(p)=\Sigma_{\varepsilon \in p} c_{e}$. Finding a static flow satisfying (1)-(4) is equivalent to solving the following primal linear programme:

$$
\begin{aligned}
& \min \sum_{p \in P^{T}} c(p) x_{p} \\
& \text { subject to } \sum_{p \in P^{T}} x_{p} \geq \frac{D}{T} \\
& \sum_{e \in A_{a}^{B}} \frac{1}{u_{e}}\left(\sum_{p \in P^{T}: e \in p} x_{p}\right) \leq 1 \quad \text { for all } a \in A, \\
& x_{p} \geq 0 \quad \text { for all } p \in P^{T} .
\end{aligned}
$$

If the number of paths in $P^{T}$ and thus the number of variables in this linear programme are exponentially large, we solve the problem by duality. Now the problem became a linear programming problem which can be solved by graphical method, simplex method or any other relevant methods. Sometimes it may give fractional solutions which cannot be acceptable practically. In this case, we desire integer solutions which can be obtained by cutting plane algorithm or branch and bound method or any other relevant method. 


\section{CONCLUSION}

In this paper, we have studied the existing models of flow over times with inflow- dependent transit times which are represented by fan graph and bow graph. Together with this, it covers the important property of temporally repeated flow, inflow-preserving flow and non-existence of earliest arrival flow in it. In modified bow graph, inflow- preserving flow is turned to weakly inflowpreserving flow by convexification, which helps to change quickest weakly inflow-preserving flow into the linear programming problem. Actually flow over time problem is not only related to traffic flow problem but is applicable in many applications like evacuation planning, communication network, internet and production system.

\section{ACKNOWLEDGEMENT}

The first author acknowledges for the supports of University Grant Commission-Nepal to carry out this research work. The second and third authors thank for the support of Alexander von Humboldt Foundation.

\section{REFERENCES}

Baumann, N. and Köhler, E. 2007. Approximating earliest arrival flows with flow-dependent transit times. Discrete Applied Mathematics 155: 161-171.

Dhamala, T.N. and Pyakurel, U. 2013. Earliest arrival contraflow problem on series-parallel graphs. International Journal of Operations Research 10: 1-13.

Fleischer, L. and Skutella, M. 2002. The quickest multicommodity flow problems, in Integer programming and combinatorial optimization, In: Cook, W.J. and Schulz, A.S. (eds). Lecture Notes in Computer Science 2337: 3653.

Fleischer, L.K. and Tardos, E. 1998. Efficient continuous-time dynamic network flow algorithms. Operations Research Letters 23: 71-80.
Ford, L.R., and Fulkerson, D.R. 1958. Constructing maximum dynamic flows from static flows. Operations Research 6: 419-433.

Gale, D. 1959. Transient flows in networks. Michigan Mathematical Journal 6: 59-63.

Hall, A., Hippler, S. and Skutella, M. 2003a. Multicommodity flows over time: efficient algorithms and complexity. In: Automata, languages and programming, Baeten, J.C.M., Lenstra, J.K., Parrow, J. and Woeginger, G.J. (eds). Lecture Notes in Computer Science 2719: 397-409.

Hall, A., Langkau K. and Skutella, M. 2003b. An FPTAS for quickest multicommodity flows with inflow dependent transit times. In: Approximation, randomization and combinatorial optimization, Arora, S., Jansen, K., Rolim, J.D.P. and Sahai, A (eds), Lecture Notes in Computer Science 2764: 71-82.

Köhler, E. and Skutella, M. 2005. Flows over time with load-dependent transit times. SIAM Journal on Optimization 15: 1185-1202.

Köhler, E., Langkau K. and Skutella, M. 2002. Time-expanded graphs for flow-dependent transit times, in Algorithms-ESA 02. Lecture Notes in Computer Science 2461: 599-611.

Pyakurel, U. and Dhamala, T.N. 2015. Models and algorithms on contraflow evacuation planning network problems. International Journal of Operations Research 12: 36-46.

Pyakurel, U. and Dhamala, T.N. 2016. Continuous time dynamic contraflow models and algorithms. Advance in Operations Research 2016: Article ID 368587,7 pp.

Pyakurel, U., Nath, H.N. and Dhamala, T.N. 2018. Efficient contraflow algorithms for quickest evacuation planning. Science China Mathematics 61(11): 2089-2100. 